\title{
Cyclosporin A in idiopathic chronic fibrosing interstitial pneumonia without idiopathic pulmonary fibrosis
}

\author{
Reoto Takei ${ }^{1}$, Machiko Arita ${ }^{1}$, Fumiaki Tokioka ${ }^{1}$, Hiromasa Tachibana ${ }^{2}$, Hironobu Tokumasu ${ }^{3}$, \\ Tadashi Ishida ${ }^{1}$
}

${ }^{1}$ Department of Respiratory Medicine, Kurashiki Central Hospital, Kurashiki, Japan; ${ }^{2}$ Department of Respiratory Medicine, National Hospital Organization Minami Kyoto Hospital, Joyo, Japan; ${ }^{3}$ Department of Management, Clinical Research Center, Kurashiki Central Hospital, Kurashiki, Japan

Contributions: (I) Conception and design: R Takei, M Arita, F Tokioka; (II) Administrative support: T Ishida; (III) Provision of study materials or patients: R Takei; (IV) Collection and assembly of data: R Takei; (V) Data analysis and interpretation: H Tachibana, H Tokumasu; (VI) Manuscript writing: All authors; (VII) Final approval of manuscript: All authors.

Correspondence to: Reoto Takei, MD. Department of Respiratory Medicine, Kurashiki Central Hospital, 1-1-1 Miwa, Kurashiki, Okayama 710-8602, Japan. Email: rtakei415@gmail.com.

Background: We have sometimes observed interstitial pneumonia which had chronic course and unknown
causes but not diagnosed as idiopathic pulmonary fibrosis (IPF). However, the treatment strategy was
not established definitely. To clarify the usefulness of cyclosporin A (CsA) in idiopathic chronic fibrosing
interstitial pneumonia (iCFIP) without IPF, we examined longitudinal changes in pulmonary physiology.
Methods: Japanese patients with iCFIP without IPF treated with CsA were identified retrospectively.
Linear mixed-effects models were used to evaluate changes in pulmonary physiology after adjusting for age,
sex, and smoking history. Primary outcomes were longitudinal trajectories of the percent predicted forced
vital capacity (\%FVC), percent predicted diffusing capacity for carbon monoxide (\%DLco), and composite
physiologic index (CPI) before and after CsA.
Results: Thirty-three patients were included. Before CsA initiation, \%FVC, \%DLco, and CPI declined
at rates of $9.1 \%, 8.6 \%$ and -7.1 per 1 year, respectively. After CsA initiation, the gradient of $\%$ FVC showed
significant improvements in $0-1$ years $(6.2 \% \pm 3.0 \%$; $<0.01)$ and in $1-2$ years $(10.0 \% \pm 3.6 \%$; P $<0.01)$;
$\%$ DLco improved in $0-1$ year $(4.0 \% \pm 4.6 \%$; $=0.09)$ and in $1-2$ years $(7.0 \% \pm 5.6 \%$; $=0.02)$; and CPI
improved in $0-1$ year $(3.2 \% \pm 3.3 \% ; \mathrm{P}=0.06)$ and in $1-2$ years $(4.6 \% \pm 4.1 \% ; \mathrm{P}=0.03)$.

Conclusions: CsA for iCFIP without IPF may be associated with improvements in pulmonary physiology in 2 years. Further studies are needed to determine the role of CsA in iCFIP without IPF.

Keywords: Cyclosporin A (CsA); chronic fibrosing interstitial pneumonia (CFIP); linear mixed-effects models; pulmonary physiology

Submitted Jun 25, 2018. Accepted for publication Nov 07, 2018.

doi: $10.21037 /$ jtd.2018.11.70

View this article at: http://dx.doi.org/10.21037/jtd.2018.11.70

\section{Introduction}

Idiopathic pulmonary fibrosis (IPF) is a chronic and progressive fibrosing interstitial pneumonia and diagnosed according to 2011 American Thoracic Society/European Respiratory Society/Japanese Respiratory Society/Latin American Thoracic Association guideline (1). IPF is characterized by usual interstitial pneumonia pattern on high-resolution computed tomography (HRCT) and histopathology features (1). On the other hand, we have sometimes observed a group of interstitial pneumonia which had chronic course and unknown cause but not diagnosed as IPF. In 2013 American Thoracic Society/European Respiratory Society statement, chronic fibrosing interstitial 
pneumonia is composed of IPF and idiopathic nonspecific interstitial pneumonia (NSIP) (2). NSIP is characterized as a temporally uniform interstitial pneumonia (3). The most common abnormalities on HRCT are bilateral ground-glass opacity, irregular reticular opacities with traction bronchiectasis and bronchiolectasis, and subpleural sparing, which are helpful in distinguishing NSIP from usual interstitial pneumonia (2). The histologic features include varying amounts of interstitial inflammation and fibrosis with uniform appearances (2). Furthermore, in 2013 statement, patients who have multiple HRCT and/ or pathologic patterns are diagnosed with unclassifiable idiopathic interstitial pneumonia and some cases have chronic course (2).

The prognosis of IPF remains poor but the treatment strategies have been established gradually (1). On the other hand, the treatment strategy for idiopathic chronic fibrosing interstitial pneumonia (iCFIP) without IPF has not been established definitively. The diagnosis of iCFIP requires the exclusion of known causes of interstitial pneumonia such as environmental exposures, medication toxicity or connective tissue disease (CTD), because identification of an underlying etiology often impacts treatment and prognosis (2). Recently, the concepts of interstitial pneumonia with autoimmune features (IPAF) has been established, consists of clinical, serological and morphological features of CTD, and is used to identify features suggesting CTD but not to make a diagnosis $(2,4)$. The clinical course of IPAF has been revealed in previous studies $(5,6)$; when patients are diagnosed with IPAF, we may administer immunosuppressive therapy as a first-line treatment depending on the predisposition for CTD. Therefore, as it is substantial to distinguish IPAF from idiopathic interstitial pneumonias, we excluded IPAF from this study.

Immunosuppressive therapies have been used to treat patients with CTD-associated interstitial lung disease (ILD) (7-11). However, in patients with iCFIP, only a few previous studies reported the usefulness of immunosuppressive therapy. In a study by Johnson et al., the findings indicated a survival advantage for patients treated with cyclophosphamide and low-dose prednisolone (PSL) (12). Kondoh et al. reported that cyclophosphamide and low-dose PSL demonstrated a favorable response in fibrosing NSIP (13). In a case report, Nanki et al. reported that the addition of cyclophosphamide to PSL was useful in recovery from NSIP (14). These previous studies researched cyclophosphamide, but the usefulness of cyclosporin A (CsA), which exerts its therapeutic effect by inhibiting calcineurin $(15,16)$, has not been identified clearly. In the present study, to clarify the usefulness of CsA in patients with iCFIP without IPF, we retrospectively examined longitudinal changes in pulmonary physiology.

\section{Methods}

\section{Study population}

This retrospective cohort study was conducted at Kurashiki Central Hospital in Japan and was approved by the Ethics Committee of Kurashiki Central Hospital in accordance with the Declaration of Helsinki (approval number 2556, June $27^{\text {th }}, 2017$ ). Patients with a multidisciplinary team diagnosis of iCFIP between April 2007 and December 2014 who underwent treatment with CsA for this diagnosis were included. The diagnosis of IPF was based on 2011 American Thoracic Society/European Respiratory Society/Japanese Respiratory Society/Latin American Thoracic Association guideline (1). The diagnosis of NSIP was based on 2013 American Thoracic Society/European Respiratory Society statement and was diagnosed only by pathological features from a surgical lung biopsy (2). Specific collagen vascular disease was diagnosed by a rheumatologist. The diagnosis of IPAF was based on Fischer's criteria in 2015 (4). Clinical characteristics were ascertained from the available clinical records. The observation period ended in April of 2017. The composite physiologic index (CPI) was calculated as follows: $91.0-[0.65 \times$ percent predicted diffusing capacity for carbon monoxide $(\% \mathrm{DLco})]-[0.53 \times$ percent predicted forced vital capacity $(\% \mathrm{FVC})]+[0.34 \times$ percent predicted forced expiratory volume in 1 second (\%FEV1)] (17).

\section{$\operatorname{Cs} A$}

CsA was administrated 30 minutes before breakfast once daily (18). The initial CsA dosage was approximately $2.0-3.0 \mathrm{mg} / \mathrm{kg} /$ day. We measured blood CsA concentrations four times: before administration $\left(\mathrm{C}_{0}\right)$, and at 1,2 , and 4 hours after administration $\left(\mathrm{C}_{1}, \mathrm{C}_{2}\right.$, and $\mathrm{C}_{4}$, respectively). The area under the curve (AUC) was calculated based on these concentrations by the trapezoidal method (19). The target CsA concentration was less than $150 \mathrm{ng} / \mathrm{mL}$ for $\mathrm{C}_{0}$, the peak CsA concentration $\left(\mathrm{C}_{1}\right.$ or $\left.\mathrm{C}_{2}\right)$ was $900-1,000 \mathrm{ng} / \mathrm{mL}$, and the $\mathrm{AUC}_{0-4}$ was $1,600-2,500 \mathrm{ng} \cdot \mathrm{hour} / \mathrm{mL}$. After the patient was discharged from the hospital, we measured the $\mathrm{C}_{2}$ CsA concentration at the outpatient visit and adjusted the dosage if the $\mathrm{C}_{2}$ concentration had changed 
Table 1 Patient characteristics $(n=33)$

\begin{tabular}{|c|c|}
\hline Variables & Outcomes \\
\hline Age, years [range] & $66[60-72]$ \\
\hline Male, $\mathrm{n}[\%]$ & 20 [61] \\
\hline Ever smoker, $\mathrm{n}[\%]$ & 15 [45] \\
\hline $\mathrm{KL}-6, \mathrm{U} / \mathrm{mL}$ [range] & $1,533[1,170-1,959]$ \\
\hline SP-D, ng/mL [range] & $223[154-368]$ \\
\hline A-aDO2 (range) & $16(7.7-21.6)$ \\
\hline Desaturation of $6 \mathrm{MWT}, \mathrm{n}$ [\%] & 20 [61] \\
\hline \%FVC, \% (range) & $70.6(60.5-78.4)$ \\
\hline \%DLco, \% (range) & $50.2(36.6-60.3)$ \\
\hline CPI (range) & $46.9(36.9-57.1)$ \\
\hline Bronchoalveolar lavage, n [\%] & $31[94]$ \\
\hline Total volume of retrieved BALF, \% [range] & $57[48-61]$ \\
\hline Total cell count, /mL (range) & $0.30(0.20-0.30)$ \\
\hline Neutrophil, \% [range] & $3[1-6]$ \\
\hline Lymphocyte, \% [range] & $14[7-24]$ \\
\hline Eosinophil, \% [range] & $2[1-3]$ \\
\hline Macrophage, \% [range] & 79 [63-86] \\
\hline CD4/8 (range) & $1.41(0.84-3.35)$ \\
\hline Surgical lung biopsy, n [\%] & $17[52]$ \\
\hline Duration of CsA, weeks [range] & $61[45-178]$ \\
\hline CsA dose, mg [range] & $120[100-160]$ \\
\hline \multicolumn{2}{|l|}{ Treatment regimen } \\
\hline CsA only, n [\%] & $0[0]$ \\
\hline CsA + low-dose PSL, n [\%] & 33 [100] \\
\hline PSL dose, mg [range] & $10[10-10]$ \\
\hline CPA prior to CsA initiation, n [\%] & $7[21]$ \\
\hline Died, n [\%] & $6[18]$ \\
\hline $\begin{array}{l}\text { Cause of death, interstitial pneumonia/ } \\
\text { other }\end{array}$ & $5 / 1$ \\
\hline
\end{tabular}

KL-6, sialylated carbohydrate antigen KL-6; SP-D, surfactant protein $\mathrm{D} ; \mathrm{A}-\mathrm{aDO} 2$, alveolar-arterial oxygen difference; $6 \mathrm{MWT}$, six-minute walk test; \%FVC, percent predicted forced vital capacity; \%DLco, percent predicted diffusing capacity for carbon monoxide; CPI, composite physiologic index; BALF, bronchoalveolar lavage fluid; CsA, cyclosporine A; PSL, prednisolone; CPA, cyclophosphamide. substantially.

\section{Statistical analysis}

The longitudinal trajectories of \% FVC, \%DLco, and CPI before and after treatment with CsA were the primary outcomes. Linear mixed-effects models with random slopes and intercepts were used to evaluate changes in \% FVC, $\% \mathrm{DLco}$, and CPI, positing piecewise linear trajectories in time, with change points at the initiation of CsA treatment. All models were adjusted for potential confounders: age, sex, and smoking history. Patients who were treated with PSL up to $20 \mathrm{mg}$ /day or who were switched from another drug to CsA were included in the analysis. Based on the linear mixed-effects models for CsA, the effects of treatment on changes in lung function after 2 years of treatment were estimated as the net difference in the observed change and the counterfactual change that would have been expected if CsA treatment had not been initiated. This net treatment effect was calculated as the yearly rate of change at initiation. All statistical analyses were performed using $\mathrm{R}$ (The R Foundation for Statistical Computing V.3.4.0). Statistical significance was defined as $\mathrm{P}<0.05$.

\section{Results}

\section{Patients}

Data from 220 patients who received treatment with CsA to ILD at our hospital between April 2007 and December 2014 was obtained from the hospital's database. ILD other than iCFIP, including acute onset interstitial pneumonia $(\mathrm{n}=101)$, IPAF $(\mathrm{n}=12)$, CTD associated ILD $(n=5)$ and other disease $(n=21)$, were excluded in this study. Moreover, combined treatment with high-dose PSL over $20 \mathrm{mg} /$ day $(\mathrm{n}=22)$, insufficient lung function data $(\mathrm{n}=23)$ and discontinuation of CsA at early stage $(\mathrm{n}=3$; two had liver damage within 30 days and one had an infection within 60 days) were excluded in this study. Thus, 33 iCFIP patients treated with CsA combined with or without low-dose PSL were included in this study. The clinical characteristics of the patients are shown in Tables 1 and 2. The median age was 66 years and $20(61 \%)$ patients were men. The median \% FVC and \%DLco values were $70.6 \%$ and $50.2 \%$, respectively. The median CPI was 46.9. A high proportion 
Table 2 Relationship between radiographic and histopathological features $(\mathrm{n}=33)$

\begin{tabular}{|c|c|c|c|c|c|}
\hline HRCT feature & \multicolumn{3}{|c|}{ Histopathological feature $(n=17)$} & No SLB $(n=16)$ & Total $(n=33)$ \\
\hline Definite UIP & 0 & 1 & 0 & 0 & 1 \\
\hline Possible UIP & 0 & 2 & 1 & 3 & 6 \\
\hline Inconsistent with UIP & 2 & 8 & 3 & 13 & 26 \\
\hline
\end{tabular}

HRCT, high-resolution computed tomography; UIP, usual interstitial pneumonia; Fnsip, fibrotic nonspecific interstitial pneumonia; SLB, surgical lung biopsy.

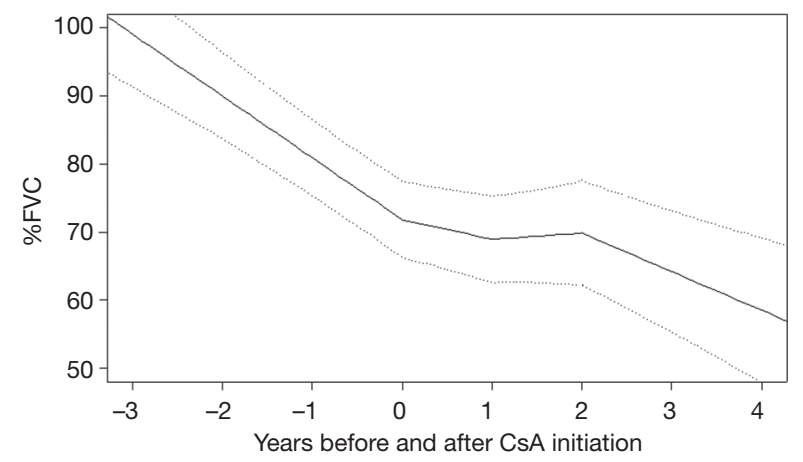

Figure 1 Longitudinal change of percent predicted forced vital capacity (\%FVC). Vertical line at time 0 denotes timing of first cyclosporin A. Solid line = mean \%FVC; broken lines show $95 \%$ confidence bands. \%FVC, percent predicted forced vital capacity.

(94\%) underwent bronchoalveolar lavage (BAL) and the median lymphocyte differential count was $14 \%$. Seventeen (52\%) patients underwent a surgical lung biopsy and 11 (33\%) patients were diagnosed with NSIP. All patients were treated with combined CsA and low-dose PSL, and the median PSL dosage was $10 \mathrm{mg} /$ day.

\section{Longitudinal change in lung function}

From the sample of 33 patients with iCFIP, there were 298 \% FVC observations and $284 \% \mathrm{DLco}$ and CPI observations used in the analysis. Before CsA initiation, \%FVC, \%DLco and CPI declined at rates of $9.1 \% \pm 2.3 \%, 8.6 \% \pm 3.3 \%$ and $-7.1 \pm 2.6$ per 1 year, respectively. In the cohort as a whole, the gradient of \% FVC showed significant improvements in $0-1$ year $(6.2 \% \pm 3.0 \% ; \mathrm{P}<0.01)$ and in $1-2$ year $(10.0 \% \pm 3.6 \%$; $\mathrm{P}<0.01$ ) from CsA initiation (Figure 1). The gradient of $\%$ DLco showed improvements in $0-1$ year $(4.0 \% \pm 4.6 \%$; $\mathrm{P}=0.09)$ and in $1-2$ years $(7.0 \% \pm 5.6 \% ; \mathrm{P}=0.02)$ (Figure 2$)$;

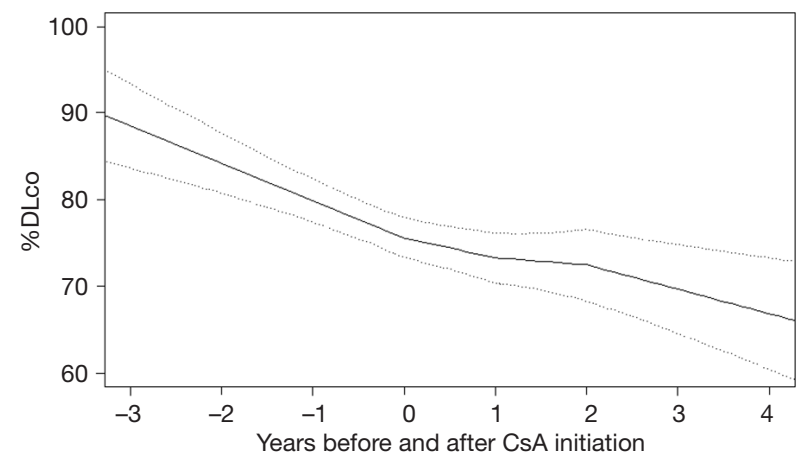

Figure 2 Longitudinal change of percent predicted diffusing capacity for carbon monoxide (\%DLco). Vertical line at time 0 denotes timing of first cyclosporin A. Solid line = mean \%DLco; broken lines show $95 \%$ confidence bands. \%DLco, percent predicted diffusing capacity for carbon monoxide.

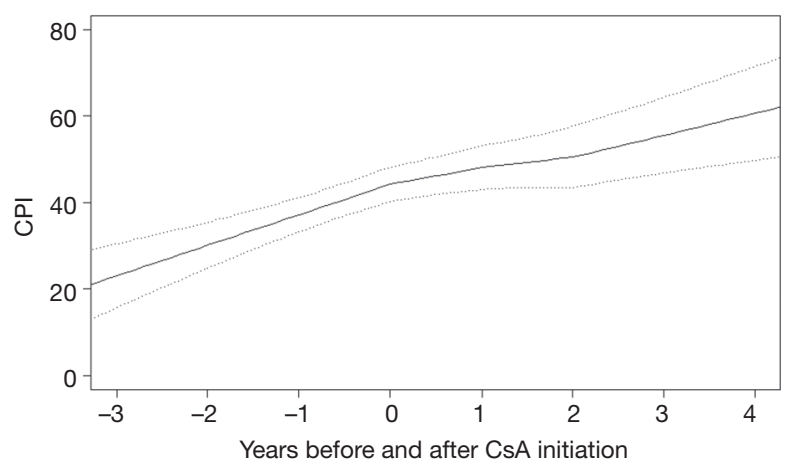

Figure 3 Longitudinal change of composite physiologic index (CPI). Vertical line at time 0 denotes timing of first cyclosporin A. Solid line = mean CPI; broken lines show 95\% confidence bands.

the gradient of CPI also showed improvements in $0-1$ year $(3.2 \% \pm 3.3 \% ; \mathrm{P}=0.06)$ and in $1-2$ years $(4.6 \% \pm 4.1 \% ; \mathrm{P}=0.03)$ (Figure 3). 


\section{Tolerability of long-term cyclosporin}

The median duration of CsA use was 61 weeks (interquartile range, 45-178 weeks) and the median daily dose of CsA was $120 \mathrm{mg}$ (interquartile range, $100-160 \mathrm{mg}$ ). CsA was well tolerated overall and was discontinued because of an adverse effect in only one patient (3.0\%). The reason for discontinuing CsA was renal dysfunction; after CsA was discontinued, the patient's renal dysfunction improved.

\section{Discussion}

In this study, we demonstrated that CsA treatment for patients with iCFIP without IPF was associated with improved \% FVC, \%DLco, and CPI values for 2 years. CsA was well tolerated with appropriate concentration adjustments. To our knowledge, this is the first study to describe the effect of CsA on the clinical course of iCFIP without IPF.

We showed the treatment of CsA in patients with iCFIP improved \% FVC, \%DLco, and CPI values for 2 years. The mechanism of CsA's action is inhibition of the action of calcineurin and completely blocking the translocation of the cytosolic component of the nuclear factor of activated $\mathrm{T}$ cells; then, this pathway inhibits the production of interleukin-2, interleukin-4, the CD40 ligand, and interferon $\gamma(15,16)$. P-glycoprotein, encoded by the multidrug resistance-1 gene and expressed on the lymphocyte surface, reduces intracellular concentrations of glucocorticoids and results in resistance to glucocorticoids (20). CsA acts antagonistically to $\mathrm{P}$-glycoprotein and improves resistance to glucocorticoids by recovering intracellular glucocorticoids levels in lymphocytes $(21,22)$. The mechanism of action of CsA on iCFIP still has not been elucidated but the immunosuppressive effects may result in improvements of iCFIP. Furthermore, in this study, all patients were treated with CsA in combination with lowdose PSL and this may have supported the effects of CsA.

It is difficult to predict the effective concentration of CsA from the dosage of CsA; the importance of measuring blood level concentrations of CsA has been reported $(18,23,24)$. The $\mathrm{C}_{0}$ value has been used as an indicator of safety, but did not necessarily correlate with the effectiveness of CsA (18). Recently, the $\mathrm{AUC}_{0-4}$ was reported to predict the effectiveness of CsA (23) and the $\mathrm{C}_{2}$ value showed a good correlation with the $\mathrm{AUC}_{0-4}(18,24,25)$. In this study, we initially measured $\mathrm{C}_{0}, \mathrm{C}_{1}, \mathrm{C}_{2}$, and $\mathrm{C}_{4}$ and adjusted the dosage of CsA by calculating the $\mathrm{AUC}_{0-4}$ to obtain an adequate concentration of CsA and avoid adverse effects. Furthermore, the concentration of CsA is unstable (24). In patients with dermatomyositis and polymyositis-associated ILD, stabilization of the CsA blood concentration was reported to be a good predictor of a therapeutic response (9). Therefore, it may be important to measure the concentration of CsA routinely. In outpatients, the measurement of only $\mathrm{C}_{2}$, which is strongly associated with the $\mathrm{AUC}_{0-4}$, was simple and minimally invasive. To obtain efficacy while avoiding an overdose of CsA, we measured $\mathrm{C}_{2}$ and adjusted the dosage if necessary. Our fine adjustments of the CsA concentration may have resulted in good efficacy and few adverse effects.

BAL provides useful information for the diagnostic evaluation of patients with suspected ILD and BAL differential cell profile frequently helps the clinician narrow the differential diagnosis in ILD. A BAL fluid lymphocyte differential count with greater than $15 \%$ indicates BAL lymphocytosis (26). We performed BAL in almost all patients (94\%) and the median BAL fluid lymphocyte differential count was $14 \%$. This result may suggest the possibility that the therapeutic responses to the corticosteroid were poor, and showed the validity of not introducing high-dose PSL treatment for the patients in this study. Furthermore, we showed good treatment responses with low-dose PSL and CsA for patients with iCFIP. CsA treatment may be indicated when a response to PSL therapy is unlikely. Prospective, randomized trials are needed to validate the effectiveness of CsA for iCFIP.

There are limitations in this study. First, it was a singlecenter retrospective study and the number of patients was small. However, we believe this was an advantage in the evaluation of patients' characteristics such as the iCFIP diagnostic process and treatment strategies including adjusting the blood concentration of CsA. Second, the outcomes included three patients who gradually decreased and discontinued CsA to avoid adverse effects from longterm CsA use over 2 years, although the effect of CsA continued. However, this factor likely worsened the results, and if it was excluded, we believe that better effects may have been demonstrated from CsA. Third, we included patients treated with PSL in combination with CsA in this study. It may contribute to the efficacy of CsA alone. However, we limited patients treated with only low-dose PSL, and we excluded patients treated with high dose PSL over $20 \mathrm{mg}$ /day. Moreover, although the efficacy of lowdose prednisolone to iCFIP has not been reported, one previous study reported that about $50 \mathrm{mg}$ of PSL was used 
to treat NSIP (27). Therefore, we though the efficacy of low-dose PSL was too limited. Last, surgical lung biopsy was only performed in $52 \%$ of the patients in this study. Needless to say, we excluded known entities associated with the development of pulmonary fibrosis such as CTD and chronic hypersensitivity pneumonitis.

\section{Conclusions}

In the present study, we reported the effectiveness of CsA and showed the possibility of CsA becoming one of the treatment methods for patients with iCFIP without IPF. Further studies are needed to determine the role of CsA in patients with iCFIP without IPF.

\section{Acknowledgements}

None.

\section{Footnote}

Conflicts of Interest: $\mathrm{T}$ Ishida has received honoraria from Taisyo-Toyama Pharmaceutical Co., Ltd., Pfizer Japan Inc., and MSD K.K. The other authors have no conflicts of interest to declare.

Ethical Statement: The study was approved by the Ethics Committee of Kurashiki Central Hospital in accordance with the Declaration of Helsinki (approval number 2556, June $27^{\text {th }}, 2017$ ). They waived informed consent because of the retrospective nature of the medical record review.

\section{References}

1. Raghu G, Collard HR, Egan JJ, et al. An official ATS/ ERS/JRS/ALAT statement: idiopathic pulmonary fibrosis: evidence-based guidelines for diagnosis and management. Am J Respir Crit Care Med 2011;183:788-824.

2. Travis WD, Costabel U, Hansell DM, et al. An official American Thoracic Society/European Respiratory Society statement: Update of the international multidisciplinary classification of the idiopathic interstitial pneumonias. Am J Respir Crit Care Med 2013;188:733-48.

3. Katzenstein AL, Fiorelli RF. Nonspecific interstitial pneumonia/fibrosis. Histologic features and clinical significance. Am J Surg Pathol 1994;18:136-47.

4. Fischer A, Antoniou KM, Brown KK, et al. An official European Respiratory Society/American Thoracic
Society research statement: interstitial pneumonia with autoimmune features. Eur Respir J 2015;46:976-87.

5. Oldham JM, Adegunsoye A, Valenzi E, et al. Characterisation of patients with interstitial pneumonia with autoimmune features. Eur Respir J 2016;47:1767-75.

6. Ito Y, Arita M, Kumagai S, et al. Serological and morphological prognostic factors in patients with interstitial pneumonia with autoimmune features. BMC Pulm Med 2017;17:111.

7. Kurita T, Yasuda S, Amengual O, et al. The efficacy of calcineurin inhibitors for the treatment of interstitial lung disease associated with polymyositis/dermatomyositis. Lupus 2015;24:3-9.

8. Fischer A, Brown KK, Du Bois RM, et al. Mycophenolate mofetil improves lung function in connective tissue disease-associated interstitial lung disease. J Rheumatol 2013;40:640-6.

9. Shimojima $Y$, Ishii W, Matsuda $M$, et al. Effective use of calcineurin inhibitor in combination therapy for interstitial lung disease in patients with dermatomyositis and polymyositis. J Clin Rheumatol 2017;23:87-93.

10. Labirua-Iturburu A, Selva-O'Callaghan A, MartínezGómez X, et al. Calcineurin inhibitors in a cohort of patients with antisynthetase-associated interstitial lung disease. Clin Exp Rheumatol 2013;31:436-9.

11. Silver RM, Warrick JH, Kinsella MB, et al. Cyclophosphamide and low-dose prednisone therapy in patients with systemic sclerosis (scleroderma) with interstitial lung disease. J Rheumatol 1993;20:838-44.

12. Johnson MA, Kwan S, Snell NJ, et al. Randomised controlled trial comparing prednisolone alone with cyclophosphamide and low dose prednisolone in combination in cryptogenic fibrosing alveolitis. Thorax 1989;44:280-8.

13. Kondoh Y, Taniguchi H, Yokoi T, et al. Cyclophosphamide and low-dose prednisolone in idiopathic pulmonary fibrosis and fibrosing nonspecific interstitial pneumonia. Eur Respir J 2005;25:528-33.

14. Nanki N, Fujita J, Yamaji Y, et al. Nonspecific interstitial pneumonia/fibrosis completely recovered by adding cyclophosphamide to corticosteroids. Intern Med 2002;41:867-70.

15. Ho S, Clipstone N, Timmermann L, et al. The mechanism of action of cyclosporin A and FK506. Clin Immunol Immunopathol 1996;80:S40-5.

16. Schreiber SL, Crabtree GR. The mechanism of action of cyclosporin A and FK506. Immunol Today 1992;13:136-42.

17. Wells AU, Desai SR, Rubens MB, et al. Idiopathic 
pulmonary fibrosis: a composite physiologic index derived from disease extent observed by computed tomography. Am J Respir Crit Care Med 2003;167:962-9.

18. Kusaba T, Konno Y, Hatta S, et al. More stable and reliable pharmacokinetics with preprandial administration of cyclosporine compared with postprandial administration in patients with refractory nephrotic syndrome. Pharmacotherapy 2005;25:52-8.

19. Dalere GM, Lum BL, Cooney GF, et al. Comparison of three methods for cyclosporine area under the curve monitoring calculations. Ther Drug Monit 1995;17:305-7.

20. Bourgeois S, Gruol DJ, Newby RF, et al. Expression of an mdr gene is associated with a new form of resistance to dexamethasone-induced apoptosis. Mol Endocrinol 1993;7:840-51.

21. Picchianti-Diamanti A, Rosado MM, et al. P-glycoprotein and drug resistance in systemic autoimmune diseases. Int J Mol Sci 2014;15:4965-76.

22. Tanaka Y, Awazu Y, Kono K. Collagen diseases and multiple drug resistance genes. Nihon Naika Gakkai

Cite this article as: Takei R, Arita M, Tokioka F, Tachibana H, Tokumasu H, Ishida T. Cyclosporin A in idiopathic chronic fibrosing interstitial pneumonia without idiopathic pulmonary fibrosis. J Thorac Dis 2018;10(12):6695-6701. doi: 10.21037/ jtd.2018.11.70
Zasshi 2001;90:151-8.

23. Clase CM, Mahalati K, Kiberd BA, et al. Adequate early cyclosporin exposure is critical to prevent renal allograft rejection: patients monitored by absorption profiling. Am J Transplant 2002;2:789-95.

24. International Neoral Renal Transplantation Study Group. Cyclosporine microemulsion (Neoral) absorption profiling and sparse-sample predictors during the first 3 months after renal transplantation. Am J Transplant 2002;2:148-56.

25. Barakat O, Peaston R, Rai R, et al. Clinical benefit of monitoring cyclosporine $\mathrm{C} 2$ and $\mathrm{C} 4$ in long-term liver transplant recipients. Transplant Proc 2002;34:1535-7.

26. Meyer KC, Raghu G, Baughman RP, et al. An official American Thoracic Society clinical practice guideline: the clinical utility of bronchoalveolar lavage cellular analysis in interstitial lung disease. Am J Respir Crit Care Med 2012;185:1004-14.

27. Park IN, Jegal Y, Kim DS, et al. Clinical course and lung function change of idiopathic nonspecific interstitial pneumonia. Eur Respir J 2009;33:68-76. 